\title{
Intraoperative presentation of Bochdalek's hernia in an adult during robotic-assisted partial nephrectomy: An uncommon situation and literature review
}

\author{
Luca Cindolo ${ }^{1}$, Francesco Berardinelli ${ }^{1}$, Arianna Manzi ${ }^{2}$, Francesca Spagnuolo ${ }^{2}$, Elisa Fabbri ${ }^{2}$, \\ Pietro Castellan $^{1}$, Flavia Petrini ${ }^{2}$, Luigi Schips ${ }^{1}$ \\ ${ }^{1}$ Urology Dept, Robotic Unit, ASL Abruzzo 2, Italy; \\ ${ }^{2}$ UOC Anestesia Rianimazione e Terapia Intensiva, ASL Abruzzo 2, Italy.
}

\begin{abstract}
Summary Bochdalek's diaphragmatic hernia (BDH) is a congenital defect of the diaphragm that usually present during the neonatal period and rarely remain silent until adulthood.

We present a 45-year-old-female case with diagnosis of double left kidney tumor prepared for robot-assisted partial nephrectomy (RPN). During the preoperative procedure she had a reduction of inspiratory volumes and increased pulmonary pressures: the robotic camera revealed the incidental presence of the left diaphragmatic defect. We report a simultaneous nephron sparing surgery (NSS) and left posterolateral BDH correction done by the da Vinci Surgical Robot (Intuitive Surgical, Sunnyvale, CA).
\end{abstract}

KEY WORDS: Bochdalek's hernia; Diaphragmatic defect; Renal tumor; Partial nephrectomy; da Vinci robot.

Submitted 30 June; Accepted 30 September

\section{INTRODUCTION}

Congenital diaphragmatic hernia (CDH) is a developmental anomaly characterized by a failure to form a continuous sheet in order to separate the abdominal and thoracic cavities during the embryogenesis. Bochdalek's hernias (BDH) are the posterolateral hernias and they are the most frequent $(80 \%)$ with a prevalence of left side (85\%) among right side (13\%) or bilateral (2\%) (1). As a result, $\mathrm{BDH}$ represents a perinatal pathology associated with significant morbidity and mortality: infants are affected by a severe pulmonary hypoplasia and pulmonary hypertension.

$\mathrm{BDH}$ in adult is a rarely clinical entity and it remains silent until the adulthood, usually discovered incidentally, without specific symptoms or signs (2). Once diagnosed, the surgical repair is the recommended therapy and the use of laparoscopy and thoracoscopy are safe surgical approaches with low morbidity and shorter hospital stay. Even more, with the evolution of surgical technology, new approaches for repairing these defects can be developed.

We present the first report of abdominal robotic repair of left posterolateral BDH in adult done by the Da Vinci Surgical Robot (Intuitive Surgical, Sunnyvale, CA).

\section{Case report}

A 45-year-old female was admitted in our clinic after an episode of acute flank pain and the CT scan revealed a double left renal masses. She refused a preliminary renal biopsy and opted for a robot-assisted partial nephrectomy (RPN). In her medical history there was absence of abdominal or thoracic trauma or previous surgery. During physical examination we inspected no respiratory symptoms and normal abdomen. The preoperative chest X-ray, the Computed Tomography (CT) (Figure 1) and the Magnetic Resonance Imaging (MRI) (Figure2) were negative for secondary malignancy or for diaphragmatic hernia. Her hematological investigations were normal.

During the initial preoperative workout of a RPN, she undergone general anesthesia, placed in flank position and pneumoperitoneum was established up to $12 \mathrm{mmHg}$ $\mathrm{CO} 2$, nevertheless there was an immediate reduction of inspiratory volumes and an increased pulmonary pressures. Haemodynamic parameters were steadily stable as well as peripheral arterial saturation. After checking the correct placement of the tube and evaluating the depth of anesthesia, the robotic camera revealed the presence of the left posterolateral BDH: the diaphragmatic defect was measured about $5 \mathrm{~cm}$ (Figure 3). The chest was retracted and visible; the pleura and the peritoneum were "in continuum", without herniation of organs. No hernia sac was found. After a consultation with thoracic surgeon, the urologist decided to continue the procedure by a pure robot-assisted approach. The hernia was repaired with interrupted nonabsorbable sutures with Mersilene stitches and a single drainage was left in the thoracic cavity to avoid a pneumothorax (Figure 4). Then the planned RPN was done. The operative time was 170 min, the blood loss was $110 \mathrm{ml}$

The patient had an uncomplicated postoperative recovery; in the first post operative day (POD) the drain was clamped and removed in second POD. The POD 3 chest

No conflict of interest declared. 


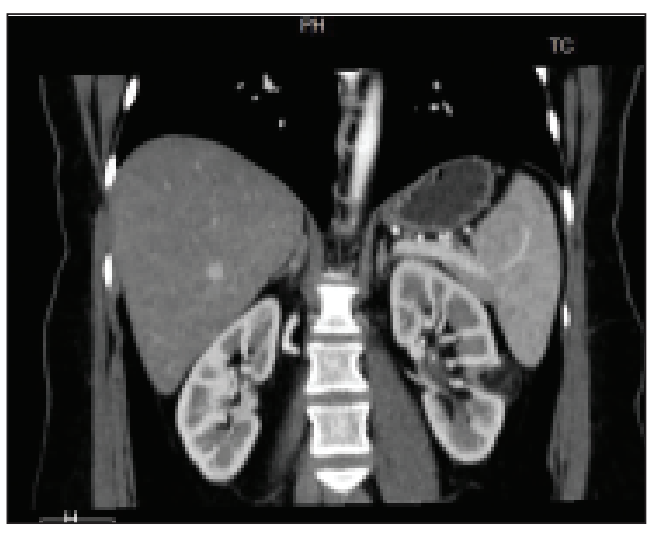

Figure 1.

CT scan showed the left kidney tumor and the absence of any visceral herniations or collapsed lung.

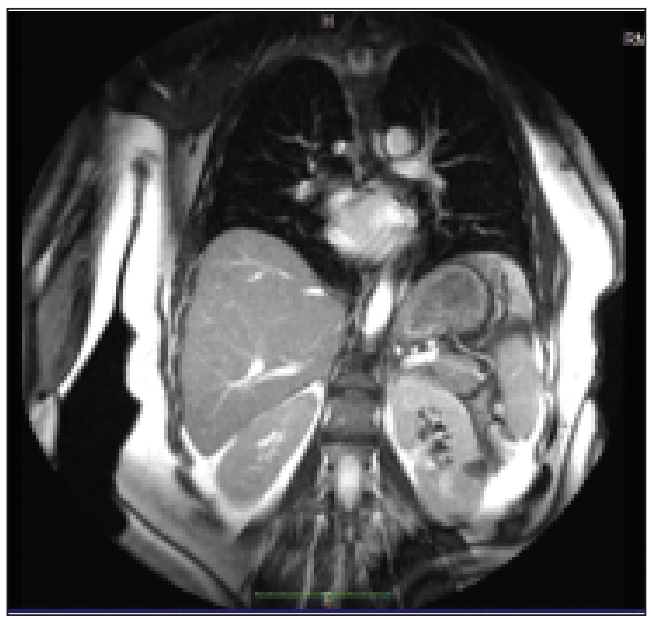

Figure 2.

RMI confirmed the diagnosis and showed no presence of the $\mathrm{BDH}$.

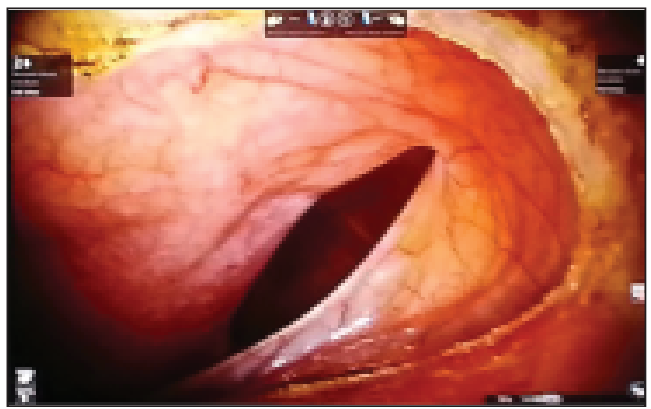

Figure 3.

Intraoperative photo of the left diaphragmatic defect.

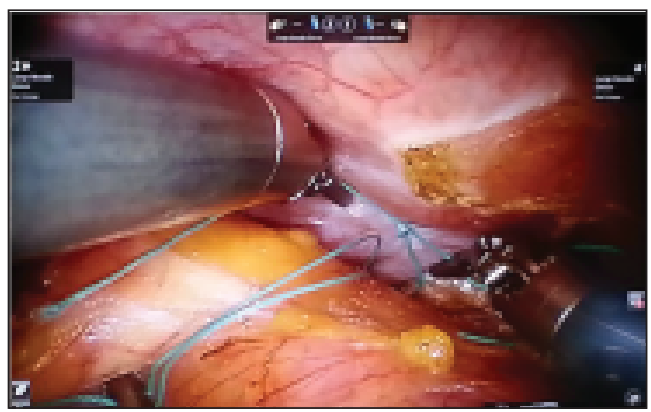

Figure 4. Intraoperative photo showing the Bochdalek diaphragmatic hernia repaired from the abdomen.

x-ray showed a fully expanded left lung with no evidence of any herniated bowel loops in the thoracic cavity. The discharge occurred in POD 4 in good general clinical conditions. The histopathology revealed a double angiomyolipoma on the left kidney $(4,1 \times 3,5 \mathrm{~cm}$ and 3,3 x 2,6 cm) with focal areas of epithelioid cells with large clear cytoplasm, absence of necrosis or atypia, low proliferative index MIB-1, and negative surgical margins. The immunophenotype was: positive for AML on the epithelioid and vascular components; positive for HMB-45; focal positive for CD117; positive for MELAN-A; positive for < 1\% Ki67; negative for AMACR and Ck7. The clinical and radiological findings at 3-month follow-up were negative.

\section{Discussion}

According to reported literature, Vincent Alexander Bochdalek first described this hernia in 1848. The exactly etiology is still under study, but however the disease is due to the failure of closure of the canal between the septum transversum and the esophagus during the $8^{\text {th }}$ week of gestation (1). BDH are the posterolateral hernias and they are the most frequent $(80 \%)$ with a prevalence of left side (85\%) among right side (13\%) or bilateral (2\%) (2). In 25$57 \%$ of cases BHD are associated with other congenital anomalies and in 10-20\% whit chromosomal disorders (3). As a result, BDH represents a perinatal pathology associated with significant morbidity and mortality: infants are affected by a severe pulmonary hypoplasia and pulmonary hypertension. BDH in adult is a rarely clinical entity and it remains silent until the adulthood, usually discovered incidentally, without specific symptoms or signs (4). In a retrospective review of 13.138 CT scans, Mullins et al. found 22 patients with incidental, asymptomatic BDH, which represents an incidence of $0.17 \%$, with a female-male ratio of $17: 5$ (77\% woman) (5).

In adults, the BDH diagnosis is difficult because of its rarity; moreover, the variety of symptoms and its asymptomatic presentation may be easily confused whit other diseases. The majority of patients present generic chronic gastrointestinal symptoms and/or respiratory symptoms related to dyspnea or breathlessness: presence of bowel sounds within the chest and the absence of breath sounds are typical findings (6). Contrariwise acute presentation of BDH include severe cardiorespiratory distress with cyanosis, tachypnea, tachycardia, abdominal pain and it's represent an emergency for the potential life threatening complications. The correct diagnosis is usually discovered as an incidental finding and the radiologic evaluation is required just to confirm. Frontal and lateral X-ray chest are the common exams performed to assess the presence of any herniations or abnormality. If the radiography is indeterminate, CT-scan and MRI offer a better visualization of the defect and help clinicians in the differential diagnosis. The management of $\mathrm{BDH}$ consist in the surgical reduction of the herniations in the abdominal cavity and the repair of the diaphragm defect as soon as possible. Traditionally, it can be performed via laparotomy and/or thoracotomy, it depends from the scenario. Recently, in order to inflicts minimal surgical trauma, thoracoscopic and laparoscopic repair of $\mathrm{BDH}$ have also been reported, with excellent results. 
Transthoracic approach allows to examine the thoracic cavity and herniated organs in detail; to avoid ischemic change, necrosis and perforation, even in the case of bilateral hernia; to separate adhesions between the contents with a good control of bleeding; to confirm the presence of lung hypoplasia and to easily reduce the hernia sac, if present (7). At the opposite, transabdominal approach is preferred for the management of the possible complications (strangulation, malrotation, perforation, peritonitis), which are documented in many $\mathrm{BDH}$, and it allows to examine the entire abdominal cavity, and easily repair the defect (8). In addition, several surgeons prefer to reinforce the suture whit some type of mesh.

A recent literature search, showed only two reports of "robotic repair of BDH". Meehan described a case report of a newborn of 37 weeks and Bethany presented a series of 8 consecutive newborns with diaphragmatic anomalies who underwent robotic repair $(9,10)$. We present the first report of patient underwent a pure robotic approach using the abdominal route and we noted that the articulating instruments offered an easy access to the posterolateral region. Moreover, referred to newborns size, we didn't have space problems and we could use the entire range of motion. We were able to close it primarily without any mesh, because there was sufficient diaphragmatic tissue to approximate.

\section{Conclusions}

In adulthood, symptomatic Bochdalek hernia is extremely rare and correct diagnosis and early treatment is significant to avoid the occurrence of morbidity and a higher complication rate. The application of robotic techniques to repair Bochdalek hernia is an excellent option because it's feasible, reduces the morbidity of surgery, allows a short hospitalization and may result in significant clinical improvement.

\section{Consent}

Written informed consent was obtained from the patient for publication of this case report and any accompanying images. A copy of the written consent is available for review by the Editor-in-Chief of this journal.

\section{REFERENCES}

1. Brown SR, Horton JD, Trivette E, et al. Bochdalek hernia in the adult: demographics, presentation, and surgical management. Hernia 2011; 15:23-30.

2. Greer, JJ. Current concepts on the pathogenesis and etiology of congenital diaphragmatic hernia. Respir Physiol Neurobiol. 2013; 189:232-40

3. Zhou Y, Du H, Che G. Giant congenital diaphragmatic hernia in an adult. J Cardiothorac Surg. 2014; 9:31.

4. Kesieme EB, Kesieme CM. Congenital diaphragmatic hernia: review of current concept in surgical management. ISRN Surgery. 2011; 2011:974041.

5. Mullins, ME, Jeffrey S, Sanjay SS, Mueller PR. Prevalence of incidental Bochdalek's hernia in a large adult population. AJR Am J Roentgenol. 2001; 177:363-66.

6. Hamid, KS, Rai SS, Rodriguez JA. Symptomatic Bochdalek hernia in an adult. JSLS. 2010; 14:279-81.

7. Tokumoto N, Tanabe K, Yamamoto H, et al. Thoracoscopic-assisted repair of a Bochdalek hernia in an adult: a case report. J Med Case Rep. 2010; 4:366.

8. Patle NM, Tantia O, Prasad P, et al. Laparoscopic repair of right sided Bochdalek hernia - a case report. Indian J Surg. 2013; 75:303-4.

9. Meehan JJ, Sandler A. Robotic repair of a Bochdalek congenital diaphragmatic hernia in a small neonate: robotic advantages and limitations. J Pediatr Surg. 2007; 42:1757-60.

10. Slater BJ, Meehan JJ. Robotic repair of congenital diaphragmatic anomalies. J Laparoendosc Adv Surg Tech A. 2009; 19:S123-7.

\author{
Correspondence \\ Luca Cindolo, MD \\ Francesco Berardinelli, MD \\ Pietro Castellan, MD (Corresponding Author) \\ castellanpietro@gmail.com \\ Luigi Schips, MD \\ Urology Dept, via S. Camillo de Lellis 1 - 66054 Vasto, Italy \\ Arianna Manzi, MD \\ Francesca Spagnuolo, MD \\ Elisa Fabbri, MD \\ Flavia Petrini, MD
}

UOC Anestesia Rianimazione e Terapia Intensiva, ASL Abruzzo 2, Italy 